\title{
The fundamental cosmic distance scale: state of the art and the Gaia perspective
}

\author{
Michael A. Dopita • Marcella Marconi • \\ Gisella Clementini · Enzo Brocato
}

Received: 10 July 2012 / Accepted: 10 July 2012 / Published online: 2 August 2012

(C) Springer Science+Business Media B.V. 2012

We are pleased to present in this Special Issue of Astrophysics \& Space Science a compilation of the latest research on the challenging issue of the cosmic distance scale. In this context, the Gaia astrometry satellite, planned for launch in the fall of 2013, will have a dramatic impact on the definition of the cosmic distance scale as it will provide trigonometric parallaxes, hence geometric distances to unprecedented accuracy of the most powerful "primary" distance indicators thus, in turn, enabling a direct re-calibration of the "secondary" indicators and a global re-assessment of the entire cosmic ladder. At the same time, due to its frequent repeated observations of the sky, Gaia will also have a significant impact revealing the "transient sky" and, most noteworthy, many $\mathrm{SNe}$ events. This volume is meant to bring together different aspects of the astronomical distance scale in order

\section{M.A. Dopita}

Research School of Astronomy and Astrophysics, Australian National University, Cotter Rd., Weston ACT 2611, Australia

M.A. Dopita

Institute for Astronomy, University of Hawaii, 2680 Woodlawn Drive, Honolulu, HI 96822, USA

M. Marconi (凶)

INAF-Osservatorio Astronomico di Capodimonte,

Via Moiariello 16, 80131 Napoli, Italy

e-mail:marcella@na.astro.it

G. Clementini

INAF-Osservatorio Astronomico di Bologna, Via Ranzani 1, 40127 Bologna, Italy

e-mail: gisella.clementini@oabo.inaf.it

\section{E. Brocato}

INAF-Osservatorio Astronomico di Roma, Via di Frascati, 33, 00040 Monteporzio Catone, Italy

e-mail: brocato@oa-roma.inaf.it to set the status of the art of the cosmic distance ladder before Gaia, discuss the extraordinary improvements expected from the mission, and, at the same time, identify preparatory work both on theoretical and empirical sides needed to allow for the full exploitation of Gaia's outcomes. The papers collected in the volume provide a reference point of the cosmic distance scale for several years to come, until the completion of the Gaia mission and the full exploitation of the Gaia catalogue.

We have organized the research material of this special issue around the following four main sections: (i) the cosmological distance ladder into context, (ii) stellar pulsation and evolution distance indicators, (iii) from local to cosmological distances to $\mathrm{H}_{0}$, (iv) Gaia: the "transient" sky and the cosmic distance ladder.

The cosmological distance ladder into context The calibration of the extragalactic distance scale leading to the final evaluation of the Hubble constant relies on geometric distance estimates for the closer stars and stellar systems. The trigonometric parallaxes measured by the Hipparcos satellite have represented a significant step forward but a much more significant improvement is expected from the results of the Gaia mission. A cornerstone in the calibration of the cosmic distance scale is represented by the distance to the Large Magellanic Cloud (LMC) and different methods have been recently adopted to reduce the uncertainty of this "first step" of the extragalactic distance ladder. The possibility to directly measure with Gaia the trigonometric parallaxes of LMC stars will definitely allow setting the distance to this crucial system.

Stellar pulsation and evolution distance indicators Several tools based on the comparison between observations and the theories of stellar evolution and stellar pulsation have been 
developed and adopted to define accurate distances within the Local Group and to provide a calibration of extragalactic distance indicators. The inter-calibration of the evolutionary and pulsation primary distance indicators is the key to reduce the systematic effects intrinsic to each individual approach and provides fundamental constraints for the derivation of an accurate extragalactic distance scale.

From local to cosmological distances to $H_{0} \quad$ Any systematic uncertainty affecting the primary distance indicators reflects in the calibration of the various types of secondary indicators. The modern view in approaching and minimising the internal uncertainties and inter-calibrations of these class of distance indicators discloses the opportunity of planning new measurements with present and next generation of ground and space facilities. At the same time, an up to date view of the most effective methods to derive the extragalactic distances represents a bridgehead in unveiling the details of physical processes at work on cosmic scale as well as the history and evolution of the Universe.

Gaia: the "transient" sky and the cosmic distance ladder The Gaia satellite will observe all objects with a magnitude in the range between 6 and $20 \mathrm{mag}$, for a total of about 1 billion targets, and will cover all the sky $\sim 70$ times on average. The capabilities of Gaia as a unique mission gathering astrometry, photometry and spectroscopy are discussed. As photometric data will be reduced and monitored in near realtime to identify transients and new sources, possibly other interesting new phenomena will be discovered. An alerts system is being implemented to promptly feed discoveries to the community and allow follow up when needed. Beyond these remarkable scientific results, some limitations either coming from the complexity of physical phenomena of the source or from the satellite itself are discussed in the volume.

We hope that the synopsis of recent results presented in this Special Issue of Astrophysics \& Space Science represents a useful picture of the state of the art for researchers and students working in the field of the cosmic distance scale or interested in this crucial problem of modern astrophysics, especially at the eve of the Gaia era.

Editor in Chief: Michael A. Dopita

Guest Editors: Enzo Brocato, Gisella Clementini \& Marcella Marconi 